\title{
Single Event Effect cross section calibration and application to quasi-monoenergetic and spallation facilities
}

Rubén García Alía, ${ }^{1,}$, Stefano Bonaldo ${ }^{2}$, Markus Brugger ${ }^{1}$, Salvatore Danzeca ${ }^{1}$, Alfredo Ferrari ${ }^{1}$, Christopher Frost ${ }^{3}$, Angelo Infantino ${ }^{1}$, Yosuke Iwamoto ${ }^{4}$, Julien Mekki ${ }^{5}$, Cris Theis ${ }^{1}$, and Adam Thornton ${ }^{1}$

1 CERN, CH-1211, Genève, Switzerland

2 RREACT Group, Dipartimento di Ingegneria Dell'Informazione, Università di Padova, 35131 Padova, Italy

3 ISIS Facility, Rutherford Appleton Laboratory, Harwell Oxford, Didcot OX11 0QX, UK

4 Japan Atomic Energy Agency (JAEA), 2-4 Shirakata, Tokai, Naka, Ibaraki 319-1195, Japan

${ }^{5}$ Centre National d'Etudes Spatiales (CNES), 18 Avenue Edouard Belin, 31400 Toulouse, France

Received: 5 January 2017 / Received in final form: 21 August 2017 / Accepted: 14 November 2017

\begin{abstract}
We describe an approach to calibrate Single Event Effect (SEE)-based detectors in monoenergetic fields and apply the resulting semi-empiric responses to more general mixed-field cases in which a broad variety of particle species and energy spectra are present. The calibration of the response functions is based both on experimental proton (30-200 MeV) and neutron (5-300 MeV) data and considerations derived from Monte Carlo simulations using the FLUKA Monte Carlo code. The application environments include the quasimonoenergetic neutrons at RCNP, the atmospheric-like VESUVIO spallation spectrum and the CHARM high-energy accelerator test facility. The agreement between the mixed-field response and that predicted through the mono-energetic calibration is within $\pm 30 \%$ for the broad variety of cases considered and thus regarded as highly successful for mixed-field monitoring applications.
\end{abstract}

\section{Introduction}

Single Event Effects (SEEs) are caused by a single, ionizing particle and though in general inducing a negative effect in electronic components, when properly calibrated can therefore be used as a means of monitoring a radiation beam or field. This paper describes the calibration process of an Single Event Upset (SEU) detector, the European Space Agency (ESA) Standard SEU Monitor [1]; and its application to the monitoring of a broad variety of mixed radiation fields.

Single event upsets (SEUs) are the change of state of a latched logic cell from one to zero or vice-versa, induced by an ionizing particle in a radiation field. An SEU is nondestructive and therefore also known as a soft-error, as the logic element can be rewritten or reset. Such events can compromise the operation of electronic components in a radiation environment, however when calibrated, the SEU response can be used to monitor the radiation levels in a broad range of applications such as space [1], high-energy accelerators [2,3] or medical physics [4]. In order to do so,

\footnotetext{
* e-mail: ruben.garcia.alia@cern.ch
}

the response of the device to be used as a detector needs to be determined through monoenergetic measurements relevant to the particle species and energy intervals present in the application mixed-field.

In addition to soft errors such as SEUs, potentially destructive (or hard) SEEs such as Single Event Latchup (SEL), a permanently state of a device whereby a parasitic thyristor structure is triggered by an ionizing particle generating a low impedance, high-current path; can also be used to monitor the characteristics of a radiation fields [5].

In a mixed radiation field (i.e. one composed of different particles species and energies) the number of SEEs $N$ in a given time period will correspond to the convolution of the individual differential particle fluences $\frac{d \phi_{i}}{d E}(E)$ and SEE cross sections $\sigma_{i}(E)$ summed over all the different particle species $i$, as expressed in equation (1):

$$
N=\sum_{i} \int \frac{d \phi_{i}}{d E}(E) \sigma_{i}(E) d E
$$

The cross sections as a function of energy can be expressed as the product of a certain constant cross section value and a weighting function $\omega_{i}(E)$ as shown in 
equation (2), therefore equation (1) can be rewritten as equation (3) where $\varphi_{i}^{e q}$ is defined as the equivalent fluence for the particle species $i$.

$$
\begin{gathered}
\sigma_{i}(E)=\sigma_{i} \cdot \omega_{i}(E) \\
N=\sum_{i} \sigma_{i} \int \frac{d \phi_{i}}{d E}(E) \omega_{i}(E) d E=\sum_{i} \sigma_{i} \phi_{i}^{e q} .
\end{gathered}
$$

It is to be noted that, whereas in equation (3) the SEE cross sections are constant and the fluxes that are multiplied by the energy-dependent weighting functions $\omega_{i}(E)$, this is only a mathematical approach to ease the detector calibration and radiation field representation. In physical terms, it is in fact the SEE cross sections that have a dependence on energy, deriving from the associated nuclear cross sections and secondary particle properties, both a function of the incident hadron energy.

For the radiation fields that will be treated in this paper, the particle species contributing to SEEs can be divided into two categories according to their interaction mechanisms: thermal neutrons and high energy hadrons (HEH), defined as hadrons above $20 \mathrm{MeV}$ plus a weighted contribution from neutrons in the $0.2-20 \mathrm{MeV}$ range [2]. Therefore, equation (2) can be expressed as equation (4); whereas equations (5) and (3) can be developed into two terms and resolved for the so-called mixed-field HEH cross section $\sigma_{H E H}^{*}$ as shown in equation (6). For a given mixedfield measurement, this value will depend on the number of measured SEEs, the associated equivalent fluences and the sensitivity to thermal neutrons. In the case of monoenergetic measurements, equation (6) takes the simple form of the ratio between the number of events and associated experimental fluence.

$$
\begin{gathered}
\sigma_{H E H}(E)=\sigma_{H E H} \cdot \omega_{H E H}(E), \\
\sigma_{t h}(E)=\sigma_{t h} \cdot \omega_{t h}(E), \\
\sigma_{H E H}^{*}=\frac{N-\sigma_{t h} \phi_{t h}^{e q}}{\phi_{H E H}^{e q}} .
\end{gathered}
$$

In the case of the thermal neutrons, SEEs are known to be induced by the neutron capture in ${ }^{10} \mathrm{~B}$ and the production of ${ }^{7} \mathrm{Li}$ and ${ }^{4} \mathrm{He}$ as ionizing products [6]. Therefore, the thermal neutron equivalent fluence can be expressed as shown in equation $(7)$ where $\omega_{t h}(E)$ is defined as shown in equation (8) and therefore $\sigma_{t h}$ defined in equation (5) corresponds to the SEE cross section at $0.025 \mathrm{eV}$.

$$
\begin{gathered}
\phi_{t h}^{e q}=\int \omega_{t h}(E) \frac{d \phi_{n}}{d E}(E) d E, \\
\omega_{t h}(E)=\left[\frac{0.025 \mathrm{eV}}{E(\mathrm{eV})}\right]^{1 / 2} .
\end{gathered}
$$

As to what concerns the HEH contribution and according to experimentally supported nuclear interaction physical considerations $[2,7,8]$, we assume that for both protons and neutrons, the respective cross sections can be expressed as shown in equation (2) where $i$ is either protons or neutrons and $\omega_{i}(E)$ is a three-parameter Weibull function as shown in equation (9) where $E_{o}$ in the onset energy and $W$ and $s$ are the scale and shape parameters, respectively. In this case the constant cross section term $\sigma_{i}$ corresponds to the saturation value.

$$
w(E)=1-e^{-\left(\left(E-E_{0}\right) / W\right)^{s}} .
$$

Therefore, the $\sigma_{H E H}^{*}$ value extracted experimentally in a mixed-field through equation (6) can be compared to the value retrieved in the monoenergetic measurement set in equation (2) which is considered as the calibrated value.

In the work presented here a set of mixed-field HEH SEE cross sections are measured and benchmarked against the calibrated value. We will use the ratio $R_{m f}$ between the mixed-field and calibrated HEH cross sections as a means of quantifying how accurately the given environment is described in terms of SEE induction.

\section{SEE cross section calibration}

The device used as a detector in the results presented in this paper is ESA Standard SEU Monitor [9], based on the AT68166F 16 Mbit Static Random Access Memory (SRAM) multi chip module from Atmel. The SRAM is designed on a $0.25 \mu \mathrm{m}$ radiation hardened process and is reported by the manufacturer to be SEL free up to a linear energy transfer (LET) value of $80 \mathrm{MeV} \mathrm{cm}^{2} / \mathrm{mg}$. In addition, it was tested up to a total ionizing dose (TID) value of $3 \mathrm{kGy}(\mathrm{Si})$ showing no degradation. The part was initially calibrated by ESA for heavy ions up to an LET of $56 \mathrm{MeV} \mathrm{cm} / \mathrm{mg}$, protons up to $230 \mathrm{MeV}$ and thermal neutrons $[1,9]$ however it needed to be re-calibrated by CERN for protons in the $30-230 \mathrm{MeV}$ range and neutrons in the $5-15 \mathrm{MeV}$ range owing to (i) the change of the core voltage of the device reported by the manufacturer in order to increase the access speed, and thus also affecting the SEU cross section (ii) the need of evaluating the response to intermediate energy neutrons $(0.2-20 \mathrm{MeV})$ which has been shown in the past [2] to have a significant impact on the high-energy accelerator SEE rate.

As will be discussed later on, the device is particularly suitable for radiation level monitoring owing to (i) its resistance to SEL and TID, (ii) its small part-to-part sensitivity spread, (iii) its relatively constant hadron cross section with energy above $\sim 20 \mathrm{MeV}$ and (iv) its capability of representing the physical distribution of the SEUs in the memory. Therefore, the monitor not only provides the measurement of the beam flux for calibrated conditions but can also be used as a means of evaluating the homogeneity and relative alignment of a beam in a test facility.

\subsection{PSI: protons between 30 and $230 \mathrm{MeV}$}

The ESA Monitor SEU cross section was measured during a set of test campaigns at the Paul Scherrer Institute (PSI) between September 2011 and April 2014. The Proton Irradiation Facility (PIF) beam line at PSI is used 
Table 1. PTB calibration for different energies in the 1-20 MeV range. Flux rates correspond to a distance of $1 \mathrm{~m}$ from the source. $\mathrm{Ti}(\mathrm{T})$ stands for tritiated titanium. The energy spread is represented by the FWHM. The relative contribution of neutrons scattered in the target is indicated by $\Phi_{s c} / \Phi$.

\begin{tabular}{llllll}
\hline Reaction & $<E_{n}>(\mathrm{MeV})$ & $F W H M_{E_{n}}(\mathrm{MeV})$ & Target & Flux $\left(/ \mathrm{cm}^{2} / \mathrm{s}\right)$ & $\Phi_{s c} / \Phi(\%)$ \\
\hline${ }^{2} H(d, n)^{3} \mathrm{He}$ & 5.0 & 0.2 & $\mathrm{D}_{2}$-gas & $5.2 \cdot 10^{3}$ & $<1.0$ \\
${ }^{2} H(d, n)^{3} \mathrm{He}$ & 8.0 & 0.2 & $\mathrm{D}_{2}$-gas & $1.9 \cdot 10^{4}$ & $<1.0$ \\
${ }^{3} H(d, n)^{4} \mathrm{He}$ & 14.8 & 0.4 & $\operatorname{Ti}(\mathrm{T})$ & $1.3 \cdot 10^{4}$ & 3.0 \\
\hline
\end{tabular}

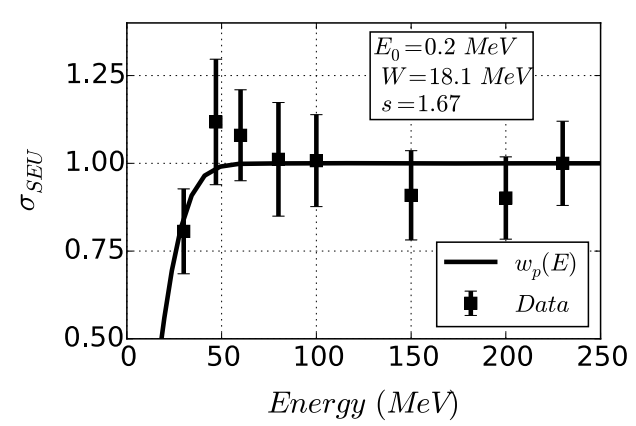

Fig. 1. Normalized PSI proton cross section data together with fitted Weibull response function.

extensively by the space community as well as by research groups in other disciplines [10]. Since 2007, the COMET cyclotron is in operation designed to produce a $1000 \mathrm{nA}$, $250 \mathrm{MeV}$ proton beam which is currently used in three Gantries and the PIF experimental station. The initial proton energy delivered by the cyclotron during the ESA Monitor calibration tests was $230 \mathrm{MeV}$, which could be degraded through the use of copper plates of different thicknesses.

At PSI, the beam intensity monitoring system consists of an ionization chamber located downstream the degrader. A plastic scintillator placed in the location of the test sample is used to measure the flux before the test runs and establish the conversion factors between the counts from the ionization chamber and the actual proton flux. The same scintillators can be used to extract the beam profile by moving it horizontally and vertically in millimeter steps. The profile is typically homogeneous within $\sim 10 \%$ in a diameter of $5 \mathrm{~cm}$. As to what regards the beam current and respective fluxes, the maximum value is roughly $5 \mathrm{nA}$, corresponding to a flux of $\sim 2 \cdot 10^{8} \mathrm{p} / \mathrm{cm}^{2} / \mathrm{s}$ at $230 \mathrm{MeV}$ and $\sim 4 \cdot 10^{7} \mathrm{p} / \mathrm{cm}^{2} / \mathrm{s}$ at $30 \mathrm{MeV}$. These values can be reduced linearly with intensity down to a lower limit of roughly $0.1 \mathrm{nA}$.

In order to evaluate the sensitivity spread among individual detectors, eight different monitors were tested at $230 \mathrm{MeV}$, yielding an average cross section value as shown in equation (10) and a relative $2 \sigma$ deviation of $7 \%$. This spread was assumed to originate from the sensitivity differences among the detectors, as the count statistics error was significantly smaller in every case (at least $5 \cdot 10^{3}$ SEU counts were accumulated during each run, corresponding to a $2 \sigma$ spread of $3 \%$ ). Moreover, this spread was assumed to be constant for the different test energies considered. When compared with the spread in commercial SRAMs calibrated for detector purposes [2,11] it can be considered as very small and typically negligible with respect other experimental uncertainties. In addition, a $10 \%$ error in the fluence value provided by the facility is assumed as typically reported.

The value and associated error reported in equation (10) are therefore the reference value for the monoenergetic HEH cross section used for the detector throughout this work.

$$
\sigma_{H E H}=(2.63 \pm 0.32) \cdot 10^{-14} \mathrm{~cm}^{2} / \mathrm{bit} .
$$

The ESA Monitor SEU cross section results were normalized to the $230 \mathrm{MeV}$ value and fitted to a threeparameter Weibull function $\omega_{p}(E)$ such as that shown in equation (9). The normalized experimental data and the respective response function $\omega_{p}(E)$ are plotted in Figure 1. In addition to the uncertainly related to the $\sigma_{H E H}$ value (deriving from the sensitivity spread and the fluence measurement) an extra $15 \%$ error is considered for the $\omega_{p}(E)$ function in order to account for the actual energy dependence of the response as opposed to the considered fit. This value corresponds to the maximum deviation of the data with respect to the fit in the considered energy interval.

\subsection{PTB: neutrons between 5 and $15 \mathrm{MeV}$}

The Physikalisch-Technishe Bundesanstalt (PTB), neutron reference fields are quasi-monoenergetic neutron reference beams in the energy range from thermal to $200 \mathrm{MeV}$ [12]. The main purpose of such beams is the energy response calibration of instruments used for neutron monitoring and dosimetry. The principle behind the production of intermediate and high energy quasi-monoenergetic fields is that of light ions (protons, deuterium) accelerated in a proton or Van-de-Graaf ( VgG) accelerator impinging on gas or solid low-Z targets (deuterium, tritium, ${ }^{7} \mathrm{Li}$ ). The reactions and field properties of the energies used in the ESA Monitor calibration campaign are shown in Table 1.

The measurements of the neutron peak fluence are performed relative to the differential n-p scattering cross section by means of a proton recoil proportional counter or a recoil proton telescope depending on the energy. Likewise, the measurement of the spectral fluence is carried out using pulsed beams and the Time-of-Flight 


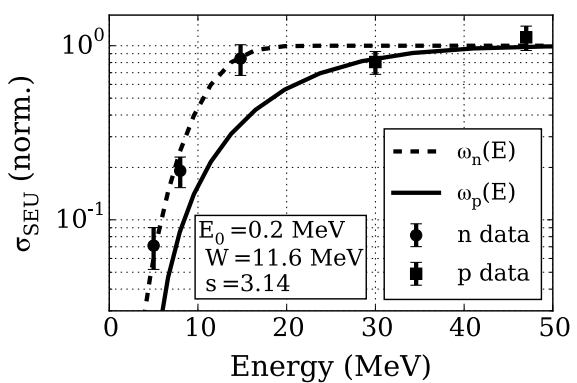

Fig. 2. Normalized PTB neutron cross section data together with the fitted neutron and proton Weibull response function for the Atmel memory.

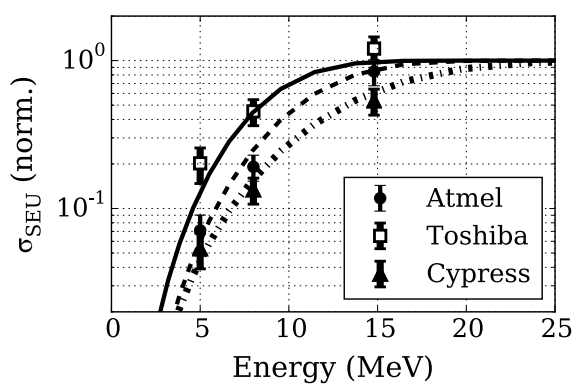

Fig. 3. PTB neutron cross section data normalized to the $230 \mathrm{MeV}$ proton value together with the fitted Weibull response function for the Atmel, Cypress and Toshiba memories. The Toshiba part was biased at $3 \mathrm{~V}$ and $5 \mathrm{~V}$ yielding compatible normalized cross sections [2], whereas and the Atmel and Cypress were biased at $3.3 \mathrm{~V}$.

(TOF) technique with scintillators and fission ionization chambers. This detection technique cannot be used for low energy neutrons at PTB $(24 \mathrm{keV}-19 \mathrm{MeV})$ due to the beam pulse frequency, therefore their spectral characterization is performed through the unfolding of Bonner sphere readings. The term low for this energy range applies to the PTB context and is related to the availability of larger quasimonoenergetic neutron energies at e.g. UCL or iThemba.

At PTB the ESA SEU Monitor was characterized with the energies reported in Table 1 . These correspond to a range in which the SEE cross section is known to have a strong dependence with energy and that can have an important impact on the overall high-energy accelerator SEE rate [2].

The response fit $w_{n}(E)$ normalized to the $\sigma_{H E H}$ value is shown in Figure 2 in logarithmic scale and along with the proton response $\omega_{p}(E)$. Similarly to the proton case, a $15 \%$ error is attributed to the use of an analytical fit $\omega_{n}(E)$ as opposed to the actual response. These results are regarded as monoenergetic for SEU calibration purposes as (i) the proportion of scattered neutrons is at the percent level, (ii) the cross section decreases rapidly with energy in this range. For the proton case, the fit in this energy interval is determined by the experimental point at $30 \mathrm{MeV}$ shown in Figure 1 and might therefore not be realistic for lower energy values. However as shown in [2], the proton (and in general charged hadron) spectrum in a high-energy accelerator mixed-field decreases strongly
Table 2. Weibull fit parameters for the different SRAMs shown in Figure 3.

\begin{tabular}{lll}
\hline SRAM & $W\left(\mathrm{MeV} \mathrm{cm}^{2} / \mathrm{mg}\right)$ & $s$ \\
\hline Cypress & 14.9 & 2.73 \\
Atmel & 11.6 & 3.14 \\
Toshiba & 9.25 & 3.02 \\
\hline
\end{tabular}

with energy in this range and therefore the impact of the proton fit on the overall response is negligible in practical terms.

In order to evaluate the impact of the SRAM feature size on the intermediate energy neutron response, Figure 3 shows the normalized cross section for the Atmel memory considered in this study together with those measure for a Toshiba ( $0.4 \mu \mathrm{m}$ feature size, reference TC554001AF-70L) and Cypress memory $(90 \mathrm{~nm}$ feature size, reference CY62157EV) both also calibrated for radiation level measurement in the high-energy accelerator environment $[2,3]$. The respective Weibull fit parameters are shown in Table 2 . As can be seen, the responses are qualitatively very similar but visibly differ among the different components.

The comparison of the response of different SRAM memories to intermediate energy neutrons relative to the $\mathrm{HEH}$ cross section value is relevant to the calibration procedure because it might not always be feasible to calibrate individual detector candidate references in this energy interval. Therefore, it is important to be able to evaluate the impact of the actual response with respect to a generalized assumption.

It is worth noting at this stage that the equivalent $\mathrm{HEH}$ (using the Toshiba response shown in Fig. 3) and thermal neutron equivalent fluxes are directly available in the FLUKA Monte Carlo code [13-15] through the HEHADEQ and THNEU-EQ generalized particles.

\section{Application to quasi-monoenergetic and spallation facilities}

\subsection{RCNP: quasi-monoenergetic neutrons at $\mathbf{1 0 0}$ and $300 \mathrm{MeV}$}

At the Research Center for Nuclear Physics (RCNP), quasimonoenergetic neutron beams in the $80-400 \mathrm{MeV}$ range are provided through the ${ }^{7} \operatorname{Li}(p, x n)$ reaction [16]. A $1 \mathrm{~cm}$ thick enriched Li target is used to produce the neutrons. An NE213 liquid scintillator was used to detect the generated neutron spectra using a TOF technique. The proton energy during the ESA Monitor calibration experiments performed in November 2014 was determined to be 100 and $296 \mathrm{MeV}$, yielding neutron peak energies of 96 and $293 \mathrm{MeV}$ respectively. As a figure-of-merit of the contribution of scattered neutrons to the total spectrum, the ratio between the peak and total above $3 \mathrm{MeV}$ fluences is used, $\Phi_{\text {peak }} / \Phi_{>3 \mathrm{MeV}}$, which was reported to be equal to 0.41 for $100 \mathrm{MeV}$ and 0.44 for $296 \mathrm{MeV}$. The relatively large proportion of off-peak neutron justifies the treatment of the quasi-monoenergetic neutron beams as a mixed-field case in which results or assumptions of the monoenergetic response need to be applied in order to 


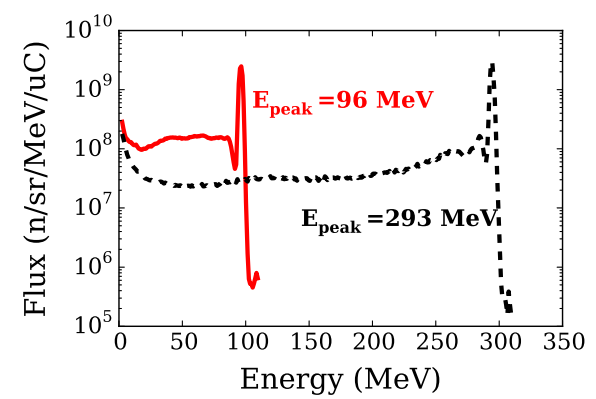

Fig. 4. RCNP spectra for 100 and $296 \mathrm{MeV}$ as measured through the NE213 TOF technique [16].

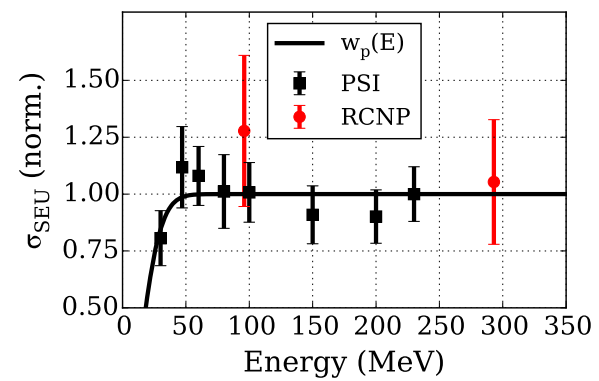

Fig. 5. Normalized RCNP neutron quasi-monoenergetic cross section data together with the fitted Weibull proton response function.

retrieve the cross section value for the energy of interest. The beam intensity is monitored using a Faraday cup collecting the protons after interacting with the production target.

The measured spectra as reported by the facility are shown in Figure 4. The response function $\omega_{n}(E)$ is convoluted with the neutron fluxes $\frac{d \phi_{n}}{d E}(E)$ in order to yield the $\phi_{H E H}^{e q}$ from equation (3) in which the thermal neutron contribution is assumed to be negligible. The resulting expression is shown in equation (11). In addition and similarly to what is performed in [17], one can also consider the extreme cases in which the quasi-monoenergetic SEE cross section is derived based (i) only on the neutron peak fluence $\Phi_{p e a k}$, and represented as $\sigma_{n p}$, and (ii) the full neutron spectrum, and represented as $\sigma_{n}$.

$$
\sigma_{H E H}^{*}=\frac{N}{\int w_{n}(E) \frac{d \phi_{n}(E)}{d E} d E} .
$$

The resulting cross section values of the two different energies and three expressions considered are shown in Table 3. Two important conclusions can be drawn from the relative comparison of the different cross sections. The fact that the cross section considering the weighted response $\left(\sigma_{H E H}^{*}\right)$ is less than $10 \%$ larger than the one considering the total neutron flux $\left(\sigma_{n}\right)$ indicates that the impact of the offpeak neutrons in the SEU induction is similar to that associated to the peak itself. Therefore, the consideration of only the latter in the cross section derivation (i.e. the $\sigma_{n p}$ ) can lead to a significant overestimation.

It is to be noted at this stage however that this situation would not apply to lower quasi-monoenergetic energies or response functions $w(E)$ with a larger energy dependence, for which the difference between $\sigma_{H E H}^{*}$ and $\sigma_{n}$ could be significant.
Table 3. SEU cross section summary for the ESA SEU Monitor at RCNP. The associated relative $2 \sigma$ uncertainties are $26 \%$, as justified in the text.

\begin{tabular}{|c|c|c|c|}
\hline $\begin{array}{l}\text { Energy } \\
(\mathrm{MeV})\end{array}$ & $\left(\cdot \sigma_{H E H_{14}}^{*} \mathrm{~cm}^{2} / \mathrm{bit}\right)$ & $\begin{array}{l}\sigma_{n} \\
\left(\cdot 10^{-14} \mathrm{~cm}^{2} / \mathrm{bit}\right)\end{array}$ & $\begin{array}{l}\sigma_{n p} \\
\left(\cdot 10^{-14} \mathrm{~cm}^{2} / \mathrm{bit}\right)\end{array}$ \\
\hline 96 & 3.36 & 3.24 & 7.29 \\
\hline 293 & 2.77 & 2.59 & 6.37 \\
\hline
\end{tabular}

In order to compare them with the high energy proton case, the resulting quasi-monoenergetic RCNP cross sections considered are those labeled as $\sigma_{H E H}^{*}$ in Table 3. These values have an uncertainty deriving from the count statistics, the sensitivity spread, a $10 \%$ margin in the neutron flux measurement and a $15 \%$ uncertainty related to the use of $w_{n}(E)$ as a response function. The normalized cross section results are plotted in Figure 5. As can be seen, the 96 and $293 \mathrm{MeV}$ neutron values are $27 \%$ and $5 \%$ larger than the saturation value, respectively; and therefore within the considered statistical uncertainty. This is compatible with the fact that in virtue of their similar nuclear reaction cross section protons and neutrons are expected to yield equivalent SEE cross sections above roughly $50 \mathrm{MeV}[18,19]$.

Despite being compatible with the expected saturation value, the normalized quasi-monoenegetic neutron cross section at $96 \mathrm{MeV}$ is clearly larger than that obtained at $293 \mathrm{MeV}$ and, as will later be shown, than those obtained at other mixed-field facilities. It is to be noted that other experiments performed during the same test campaign showed a similar trend, therefore a systematic error in the associated dosimetry cannot be excluded.

\subsection{VESUVIO: atmospheric-like neutron spectrum}

The VESUVIO neutron beam is part of the ISIS-STFC laboratory in Oxford, UK. Despite its main use as a condensed matter research instrument [20] benchmark measurements have been performed proving that VESUVIO provides a neutron spectrum similar to the ambient at sea level. Neutrons are generated through the interaction of a $800 \mathrm{MeV}, 2 \mu \mathrm{A}$ proton beam with a tungsten spallation target. The proton beam is accelerated in a synchrotron as two $100 \mathrm{~ns}$ long pulses with a frequency of $50 \mathrm{~Hz}$. The VESUVIO beamline is at $60^{\circ}$ with respect to the initial proton beamline. The neutron flux obtained above $10 \mathrm{MeV}$ is $\sim 5.8 \cdot 10^{4} \mathrm{n} / \mathrm{cm}^{2} / \mathrm{s}$ therefore roughly an order of magnitude lower than those available at TRIUMF or LANSCE. The neutron spectrum is calculated using the MCNPX Monte Carlo simulation tool and benchmarked against TOF measurements performed with different detectors including Bonner spheres, activation foils, CCD devices and thin film breakdown counters [20]. The flux measurement during the VESUVIO experiments relies on the benchmarked MCNPX calculations scaled with the beam current.

Concerning the ESA SEU Monitor measurements performed in March 2014, two configurations were used: one with the primary neutron VESUVIO beam and one with a $1.5 \mathrm{~mm} \mathrm{Cd}$ foil surrounding the detector in order to 


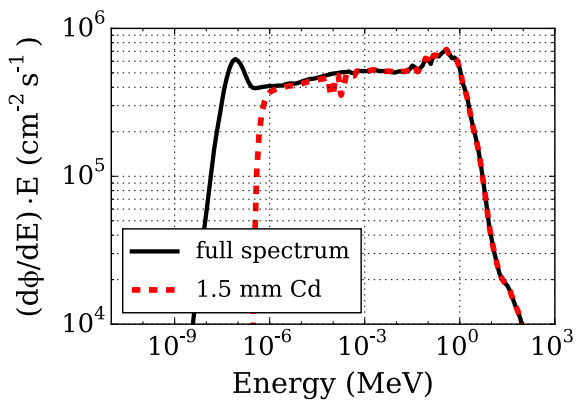

Fig. 6. VESUVIO lethargy flux spectrum with and without the Cadmium absorber.

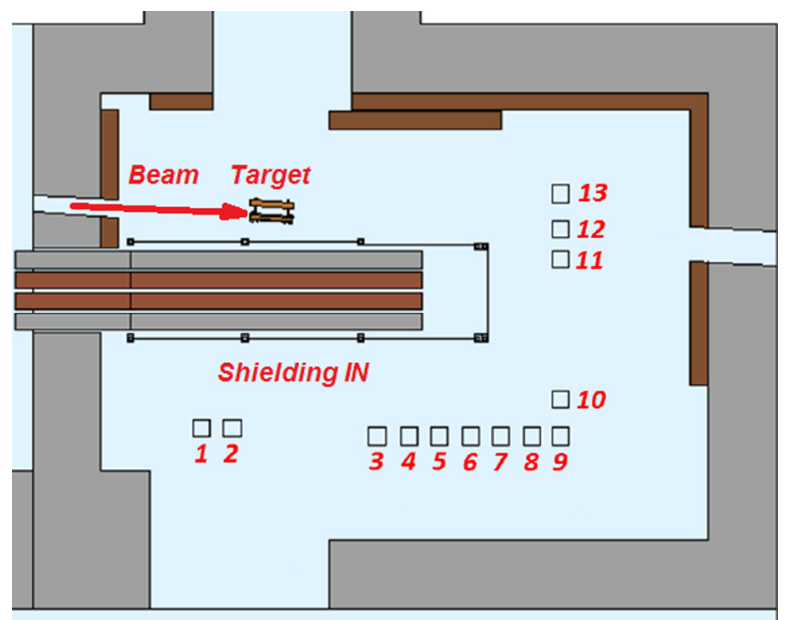

Fig. 7. FLUKA CHARM model horizontal cut at target level.

absorb the thermal neutrons by means of the roughly $7000 \mathrm{~b}$ associated capture cross section. The respective spectra can be seen in Figure 6 as reported by the facility through benchmarked MCNPX simulations.

As the ESA Monitor is known to be sensitive to thermal neutrons and provided the high energy neutron spectrum remains unaltered when introducing the cadmium absorber, the thermal neutron SEU cross section $\sigma_{t h}$ can be extracted through the ratio of the differences of the SEU rates (SER) and the respective equivalent thermal neutron fluxes $\phi_{t h}^{e q}$, without and with absorber, as shown in equation (12).

$$
\begin{aligned}
\sigma_{t h} & =\frac{S E R_{f u l l}-S E R_{C d}}{\phi_{t h}^{e q(f u l l)}-\phi_{t h}^{e q(C d)}} \\
& =(2.30 \pm 0.62) \cdot 10^{-15} \mathrm{~cm}^{2} / \mathrm{bit}
\end{aligned}
$$

The HEH cross section can therefore be derived from equation (3) yielding the result shown in equation (13):

$$
\sigma_{H E H}^{*}=(2.79 \pm 0.60) \cdot 10^{-14} \mathrm{~cm}^{2} / \mathrm{bit} .
$$

When divided by $\sigma_{\mathrm{HEH}}^{*}$ the resulting ratio is $1.06 \pm 0.27$ therefore the VESUVIO atmospheric-like spectrum cross section value is fully compatible with the monoenergetic calibration.

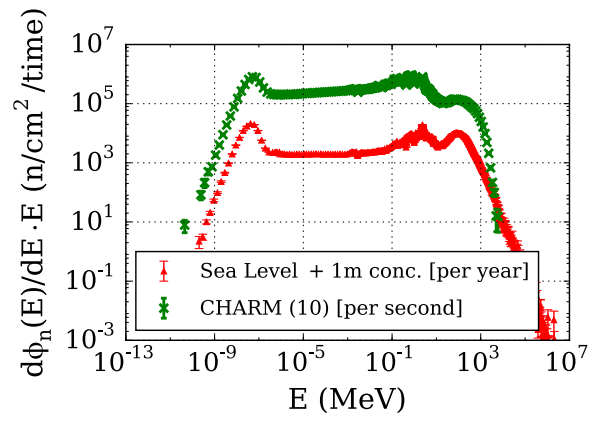

Fig. 8. Simulated neutron spectra in lethargy form.

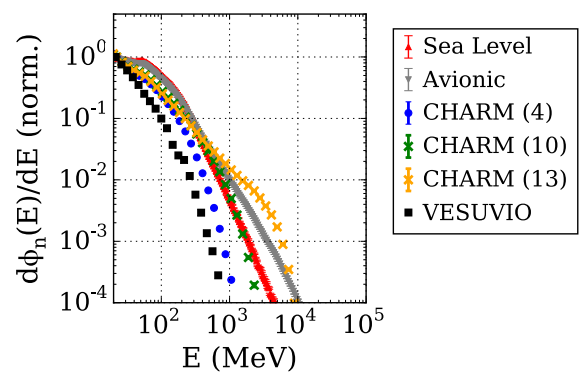

Fig. 9. Simulated neutron spectra above $20 \mathrm{MeV}$ normalized to their value at $20 \mathrm{MeV}$.

\subsection{CHARM: high-energy accelerator like mixed-field}

SEU measurements on the ESA Reference Monitor were performed in the CHARM (Cern high-energy accelerator mixed field) facility at CERN. As further described in [5,21], a mixed-field radiation environment similar to that encountered in the high-energy accelerator context is generated through the interaction of a $24 \mathrm{GeV}$ proton beam extracted from the Proton Synchrotron (PS) LHC injector with a $50 \mathrm{~cm}$ metallic target. Different target, shielding and test location configurations are available in order to yield a broad range of radiation field intensities, compositions and spectra for a given fixed proton intensity on the target. The facility radiation levels are simulated using the FLUKA Monte Carlo code and benchmarked against measurements with the RadMON system [22]. In both cases the radiation levels are normalized to the protons on target, which are measured using a Secondary Emission Counter (SEC) calibrated through regular aluminum activation experiments.

A horizontal cut of the FLUKA geometry of the facility at the target level can be seen in Figure 7. In the figure, the four $20 \mathrm{~cm}$ layers of shielding (concrete on the outside, iron on the inside) are placed inside the irradiation area, but can be remotely extracted. In addition, the 13 possible test locations are indicated in red and represented through a number in brackets in the plots and tables below. Moreover, it is to be noted that though the shielding is placed inside the facility in Figure 7 this is only for illustration purposes, as the test results and radiation field values shown in this paper all correspond to situations in which the movable shielding was kept outside of the irradiation room. 
Table 4. Equivalent HEH mixed-field cross section results for different environments and ratio relative to the monoenergetic value in brackets.

\begin{tabular}{|c|c|c|c|c|c|}
\hline Environment & HEH comp. & R-factor & $\frac{\Phi_{H E H e q}}{\Phi_{H E H}}$ & $H_{10 \%}(\mathrm{MeV})$ & $\sigma_{H E H}\left(\mathrm{~cm}^{2} / \mathrm{bit}\right)$ \\
\hline VESUVIO & $100 \% n$ & 29 & 2.32 & 150 & $\begin{array}{l}2.79 \cdot 10^{-14} \\
(1.06)\end{array}$ \\
\hline $\begin{array}{l}\text { CHARM, } \\
\text { Al-low target, } \\
1 \text {, no shield. }\end{array}$ & $\begin{array}{l}71 \% n \\
12 \% p \\
17 \% \pi^{ \pm}\end{array}$ & 0.45 & 1.50 & 190 & $\begin{array}{l}2.67 \cdot 10^{-14} \\
(1.03)\end{array}$ \\
\hline $\begin{array}{l}\text { CHARM, } \\
\text { Cu target, } \\
\text { 4, no shield. }\end{array}$ & $\begin{array}{l}71 \% n \\
14 \% p \\
15 \% \pi^{ \pm}\end{array}$ & 1.24 & 1.30 & 360 & $\begin{array}{l}2.75 \cdot 10^{-14} \\
(1.06)\end{array}$ \\
\hline $\begin{array}{l}\text { CHARM, } \\
\text { Al-low target, } \\
\text { 13, no shield. }\end{array}$ & $\begin{array}{l}26 \% n \\
51 \% p \\
19 \% \pi^{ \pm}\end{array}$ & & 1.05 & $3.8 \mathrm{GeV}$ & $\begin{array}{l}3.95 \cdot 10^{-14} \\
(1.50)\end{array}$ \\
\hline
\end{tabular}

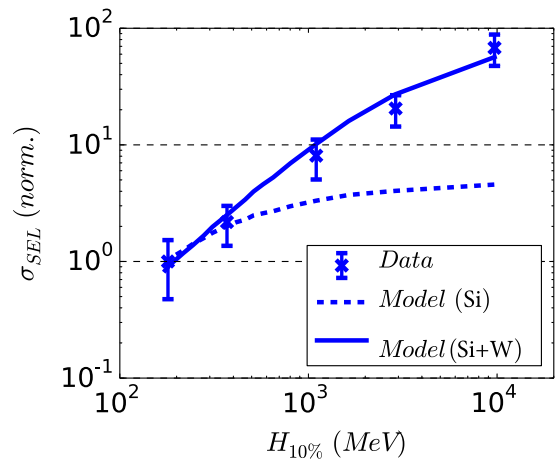

Fig. 10. Simulated and experimental SEL cross section for a commercial SRAM with a high-Z dominated response as shown in [5].

The particle energy spectra are simulated using the FLUKA code. The neutron spectrum at test location 10 (copper target, no shielding) is shown in Figure 8 in lethargy form together with the ground-level neutron spectrum as simulated in [23] using FLUKA for Vancouver, Canada $\left(49^{\circ} 15^{\prime} 0^{\prime \prime} N 123^{\circ} 8^{\prime} 0^{\prime \prime} W\right.$, sea level plus $1 \mathrm{~m}$ concrete propagation in order to allow for some neutron thermalisation).

In addition, the high-energy neutron spectra for various CHARM locations, VESUVIO facility and atmospheric cases (ground level and $12 \mathrm{~km}$ altitude, both for the Vancouver coordinates introduced above) are shown in Figure 9 normalized to the $20 \mathrm{MeV}$ value. In the CHARM mixed-field environment it is to be reminded however that other hadrons are present in addition (notably protons and pions) which will be similarly efficient in inducing SEEs and which typically have harder spectra than neutrons. As can be seen, whereas the VESUVIO and CHARM(4) spectra are significantly softer than the atmospheric ones, the CHARM(10) follows the two latter up to roughly $1 \mathrm{GeV}$, and the $\operatorname{CHARM}(13)$ is even harder until roughly the incident proton beam energy at CHARM $(24 \mathrm{GeV})$. In order to quantify the hardness of each spectrum, the $10 \%$ hardness factor $\left(H_{10 \%}\right)$ is defined as the energy above which $10 \%$ of the $\mathrm{HEH}$ spectrum remains. The $H_{10 \%}$ value for CHARM(10) is $790 \mathrm{MeV}$, whereas for the ground level and avionics spectra shown in Figure 9, it is 380 and $830 \mathrm{MeV}$ respectively.

Results for the CHARM mixed-field ESA reference SEU measurements are shown in Table 4 together with the VESUVIO measurements. The CHARM R-factors were experimentally obtained using the technique of applying two different voltages on the RadMON SRAM detector [24]. As can be seen, despite the relatively large R-factor in VESUVIO and large intermediate energy neutron impact (quantified through the $\Phi_{H E H e q} / \Phi_{H E H}$ ratio) the results for the three first environments in the table (with $10 \%$ hardness values below $400 \mathrm{MeV}$ ) are highly compatible with the monoenergetic HEH cross section, $\sigma_{H E H}^{*}$. This is due to the fact that the environments are well described through the monoenergetic calibration introduced in Section 3. However, for test location 13, the mixed-field $\mathrm{HEH}$ cross section is a factor 1.5 larger than the monoenergetic one. This is interpreted as the effect of the very energetic spectrum $\left(H_{10 \%}=3.8 \mathrm{GeV}\right)$ and the expected increase of the ESA reference monitor SEU cross section in the $200 \mathrm{MeV}-3 \mathrm{GeV}$ range [25]. The relatively moderate increase is due to the saturated nuclear reaction cross sections in silicon in this energy range combined with a similar high-LET fragment production yield. However, the generation of light fragments (e.g. alphas) to which the ESA reference monitor is sensitive to increases by a factor $\sim 3$ in the range specified above.

In contrast, as shown in Figure 10 (adapted from [5]), for a part with an SEL cross section dominated by high-Z material fragments, the HEH cross section increase at CHARM between an $H_{10 \%}$ value of $100 \mathrm{MeV}$ and $1 \mathrm{GeV}$ is roughly a factor 10, thus exhibiting a much larger 
sensitivity than that obtained for the ESA SEU Monitor studied in this paper. Therefore, applying test results from soft spectrum experimental conditions such as VESUVIO or CHARM(1) can lead to significant underestimations of the application SEE rate $\left(12 \mathrm{~km}\right.$ altitude, $\left.H_{10 \%}=800 \mathrm{MeV}\right)$ or LHC tunnel $\left(H_{10 \%}=2 \mathrm{GeV}\right)$.

\section{Summary and conclusions}

Results for the monoenergetic calibration of the ESA SEU Monitor are shown and applied to various mixed-field cases. For a broad range of test environments (100 and $300 \mathrm{MeV}$ quasi-monoenergetic neutron fields at RCNP, spallation neutron spectrum at VESUVIO and soft CHARM mixed-fields) the figure-of-merit defined as the ratio between the mixed-field and reference HEH cross sections is compatible with 1 . In order to achieve this value, the thermal and intermediate energy neutron responses need to be carefully considered through the sensitivity calibration and spectrum simulation or measurement. Therefore, this method provides both a general approach and specific calibration results that can be used to determine the degree of accuracy in which a mixed radiation field is described in terms of capability of inducing SEEs. For quasi-monoenergetic and spallation neutron sources, as well as for soft spectra in an accelerator mixed-field, the approach is highly satisfactory, fully meeting the respective monitoring needs in terms of accuracy and precision.

However, for harder mixed-field cases such as those obtained in the CHARM longitudinal positions (e.g. 13) the response function up to $200 \mathrm{MeV}$ is typically not enough to account for the possible cross section increase with energy. This is particularly the case for components with high- $Z$ materials near their sensitive volumes and relatively high LET onsets and therefore highlights the importance of considering such dependence (either experimentally or through simulations) for applications in high energetic hadron environments (e.g. avionics or the LHC tunnel).

Future work will be devoted to further combining experimental results and models in order to more accurately represent the SEE response of detector candidates, focusing especially on the thermal and intermediate $(0.2-20 \mathrm{MeV})$ neutron range, as well as in the response at energies above typical medical proton cyclotron facilities $(\sim 200 \mathrm{MeV})$, still relevant for the avionic and high-energy accelerator environment.

\section{References}

1. R. Harboe-Sorensen, C. Poivey, F.X. Guerre, A. Roseng, F. Lochon, G. Berger, W. Hajdas, A. Virtanen, H. Kettunen, S. Duzellier, IEEE Trans. Nucl. Sci. 55, 3082 (2008)

2. K. Roed, M. Brugger, D. Kramer, P. Peronnard, C. Pignard, G. Spiezia, A. Thornton, IEEE Trans. Nucl. Sci. 59, 1040 (2012)
3. S. Danzeca, G. Spiezia, M. Brugger, L. Dusseau, G. Foucard, R.G. Alia, P. Mala, A. Masi, P. Peronnard, J. Soltes et al., IEEE Trans. Nucl. Sci. 61, 3458 (2014)

4. K.S. Ytre-Hauge, A. Velure, E.F. Larsen, C.H. Stokkevåg, D. Röhrich, N. Brekke, O.H. Odland, Nucl. Instrum. Methods Phys. Res. Sect. A: Accel. Spectrom. Detect. Assoc. Equip. 804, 64 (2015)

5. R.G. Alia, M. Brugger, S. Danzeca, V. Ferlet-Cavrois, C. Frost, R. Gaillard, J. Mekki, F. Saigne, A. Thornton, S. Uznanski et al., IEEE Trans. Nucl. Sci. 62, 2555 (2015)

6. R.C. Baumann, E.B. Smith, Neutron-induced boron fission as a major source of soft errors in deep submicron SRAM devices, in Reliability Physics Symposium, 2000, Proceedings. 38th Annual 2000 IEEE International (2000), pp. 152 157

7. B. Sierawski, K. Warren, R. Reed, R. Weller, M. Mendenhall, R. Schrimpf, R. Baumann, V. Zhu, Contribution of lowenergy $(<10 \mathrm{MeV})$ neutrons to upset rate in a $65 \mathrm{~nm}$ SRAM, in Reliability Physics Symposium (IRPS), 2010 IEEE International (2010), pp. 395-399

8. S.I. Abe, Y. Watanabe, IEEE Trans. Nucl. Sci. 61, 3519 (2014)

9. R. Harboe-Sorensen, F.X. Guerre, A. Roseng, Design, testing and calibration of a reference seu monitor system, in 8th European Conference on Radiation and Its Effects on Components and Systems, 2005. RADECS 2005 (2005) pp. B31

10. W. Hajdas, F. Burri, C. Eggel, R. Harboe-Sorensen, R. de Marino, Radiation effects testing facilities in PSI during implementation of the Proscan project, in Radiation Effects Data Workshop, 2002 IEEE (2002), pp. 160-164

11. S. Danzeca, J. Cesari, M. Brugger, L. Dusseau, A. Masi, A. Pineda, G. Spiezia, IEEE Trans. Nucl. Sci. 61, 3451 (2014)

12. S. Röttger, R. Böttger, F. Brooks, A. Buffler, J. Meulders, R. Nolte, F. Smit, F. Wissmann, The PTB neutron reference fields (PIAF)-quasi-monoenergetic neutron reference fields in the energy range from thermal to $200 \mathrm{MeV}$, in 4th International Workshop on Nuclear Fission and FissionProduct Spectroscopy (AIP Publishing, 2009), Vol. 1175, pp. 375-381

13. A. Ferrari, P.R. Sala, A. Fasso, J. Ranft, CERN-2005-102005, INFN/TC-05/11, SLAC-R-773 (2005)

14. T. Böhlen, F. Cerutti, M. Chin, A. Fassò, A. Ferrari, P. Ortega, A. Mairani, P. Sala, G. Smirnov, V. Vlachoudis, Nucl. Data Sheets 120, 211 (2014)

15. G. Battistoni, T. Boehlen, F. Cerutti, P.W. Chin, L.S. Esposito, A. Fassò, A. Ferrari, A. Lechner, A. Empl, A. Mairani et al., Ann. Nucl. Energy 82, 10 (2015)

16. Y. Iwamoto, M. Hagiwara, D. Satoh, S. Araki, H. Yashima, T. Sato, A. Masuda, T. Matsumoto, N. Nakao, T. Shima et al., Nucl. Instrum. Methods Phys. Res. Sect. A: Accel. Spectrom. Detect. Assoc. Equip. 804, 50 (2015)

17. D. Lambert, J. Baggio, G. Hubert, P. Paillet, S. Girard, V. Ferlet-Cavrois, O. Flament, F. Saigne, J. Boch, B. Sagnes et al., IEEE Trans. Nucl. Sci. 53, 1890 (2006)

18. H.H.K. Tang, IBM J. Res. Dev. 40, 91 (1996)

19. D. Lambert, F. Desnoyers, D. Thouvenot, Investigation of neutron and proton SEU cross-sections on SRAMs between a few $\mathrm{MeV}$ and $50 \mathrm{MeV}$, in 2009 European Conference on Radiation and Its Effects on Components and Systems (RADECS) (2009) pp. 148-154 
20. C. Andreani, A. Pietropaolo, A. Salsano, G. Gorini, M. Tardocchi, A. Paccagnella, S. Gerardin, C.D. Frost, S. Ansell, S.P. Platt, Appl. Phys. Lett. 92, 114101 (2008)

21. J. Mekki, M. Brugger, R.G. Alia, A. Thornton, N.C.D.S. Mota, S. Danzeca, IEEE Trans. Nucl. Sci. 63, 2106 (2016)

22. G. Spiezia, P. Peronnard, A. Masi, M. Brugger, M. Brucoli, S. Danzeca, R.G. Alia, R. Losito, J. Mekki, P. Oser et al., IEEE Trans. Nucl. Sci. 61, 3424 (2014)
23. A. Infantino, E.W. Blackmore, M. Brugger, R.G. Alía, M. Stukel, M. Trinczek, Nucl. Instrum. Methods Phys. Res. Sect. A: Accel. Spectrom. Detect. Assoc. Equip. 838, 109 (2016)

24. D. Kramer, M. Brugger, V. Klupak, C. Pignard, K. Roeed, G. Spiezia, L. Viererbl, T. Wijnands, IEEE Trans. Nucl. Sci. 58, 1117 (2011)

25. R.G. Alia, B. Biskup, M. Brugger, M. Calviani, C. Poivey, K. Roed, F. Saigne, G. Spiezia, F. Wrobel, IEEE Trans. Nucl. Sci. 60, 2469 (2013)

Cite this article as: Rubén García Alía, Stefano Bonaldo, Markus Brugger, Salvatore Danzeca, Alfredo Ferrari, Christopher Frost, Angelo Infantino, Yosuke Iwamoto, Julien Mekki, Cris Theis, Adam Thornton, Single Event Effect cross section calibration and application to quasi-monoenergetic and spallation facilities, EPJ Nuclear Sci. Technol. 4, 1 (2018) 